\title{
The Role of Formyl Peptide Receptor 1 Gene Polymorphisms in Human Colorectal Cancer
}

\author{
Shu-Qin Li ${ }^{1,2^{*}}$, Yang $\mathrm{Yu}^{1^{*}}$, Yan Zhang, ${ }^{1}$ Yan-Ping Sun ${ }^{3}$, Xin-xing Li ${ }^{3 凶}, \mathrm{Ning} \mathrm{Su}^{3 凶}$ \\ 1. School of Pharmacy, Shanghai Jiao Tong University, 800 Dongchuan Road, Shanghai, 200240, China. \\ 2. School of Pharmacy, Ruijin Hospital Affiliated to Shanghai Jiao Tong University School of Medicine, 197 Ruijin Road (No.2), Shanghai, 200025, China. \\ 3. Department of General Surgery, Shanghai Chang Zheng Hospital, Second Military Medical University, 415 Fengyang Road, Shanghai, 200003, China. \\ *These authors contributed equally to this work. \\ $\square$ Corresponding author: Department of General Surgery, Shanghai Chang Zheng Hospital, Second Military Medical University, 415 Fengyang Road, Shanghai, \\ 200003, China. Tel: +86 2181885599; Fax: +086 2181886000. Xin-xing Li (E-mail: su4063@163.com); or Ning Su (E-mail: xingxin123456@sina.com).
}

() The author(s). This is an open access article distributed under the terms of the Creative Commons Attribution License (https://creativecommons.org/licenses/by/4.0/). See http://ivyspring.com/terms for full terms and conditions.

Received: 2019.05.05; Accepted: 2020.03.01; Published: 2020.03.25

\begin{abstract}
Formyl peptide receptor 1 (FPR1) belongs to G protein-coupled receptors expressed mainly in phagocytic leukocytes. The gene encoding FPR 1 is highly polymorphic and related to inflammation. In this study, we investigated the single nucleotide polymorphisms (SNPs) of Fprl in human colorectal cancer (CRC), and analyzed the association of Fprl SNPs with clinicopathological parameters and some specific diagnostic markers of CRC. Although the allele and genotype frequencies of Fprl SNPs in CRC tissues were not significantly different from that in whole blood cells derived from healthy Chinese subjects. Significant associations were observed between genotypes of c. $289 C>A$ and distant metastasis $(P=0.001)$, and between genotypes of c.306T $>C$ and tumor size $(P=0.016)$. Genotypes of c.546C $>A$ was closer to tumor size and lymphatic invasion $(P=0.012$ and $P=0.043$, respectively). Meanwhile, genotypes of c. $1037 \mathrm{C}>\mathrm{A}$ was related with tumor location and differentiation $(\mathrm{P}=0.000$ and $\mathrm{P}=0.005$, respectively). Besides, genotypes of $c .576 \mathrm{~T}>\mathrm{C}>\mathrm{G}$ was related with pathological type $(\mathrm{P}=0.000)$. Furthermore, several Fpr 1 SNP positions including c.289 (C>A) and c.576 (G>C>T) were related to the expression of P53 $(\mathrm{P}=0.004$ and $\mathrm{P}=0.008$, respectively), and similar results were observed between other Fprl SNP positions and CEA, HER2 and Ki-67 $(\mathrm{P}<0.05)$. Our data demonstrate that Fprl SNPs may play the important role in the progression and metastasis of CRC.
\end{abstract}

Key words: Colorectal cancer; Formyl peptide receptor 1; Single nucleotide polymorphisms; Metastasis

\section{Introduction}

Colorectal cancer (CRC) is one of the most frequently occurring malignancies in the world [1]. In 2018, CRC accounted for more than 860,000 deaths with 1.8 million new cases [1]. Several risk factors contribute to CRC progression, including advancing age, chronic intestinal inflammation, and genetic aberrations/mutations [2-4]. Current evidence suggests that genetic contribution is about 30\% [5]. Multiple genetic studies have shown that the single nucleotide polymorphisms (SNPs) are associated with the occurrence, development, metastasis and drug tolerance of CRC [6,7].

The human formyl peptide receptor 1 (FPR1) belongs to the seven-transmembrane $G$ protein- coupled receptors (GPCRs) [8]. FPR1 is known to have important functions in host defense and inflammation. It is expressed mainly on phagocytic leukocytes, neutrophils, and monocytes [8]. Binding of agonistic ligands to FPR1 results in activation of a $G$ protein-mediated signaling cascade; leading to cell chemotaxis, calcium flux, phagocytosis, and release of inflammatory factors [8-10]. FPR1 has also been detected on cells of non-hematopoietic tissues such as epithelial cells, endothelial cells, and neurons [11]. Besides, recent evidence shows that it is expressed in several types of human cancer tissues such as gastric cancer [12,13], lung cancer [14], breast cancer [15], etc. Our previous study revealed the high expression of 
FPR1 in primary human CRC tissue [16]. FPR1 mRNA expression was also associated with tumor serosal infiltration in CRC patients [16]. Human Fpr1 is a 6-kb single copy gene and located on chromosome 19 (19q13.3) [8]. Several SNPs of Fpr1 has been reported previously to be associated with blood pressure [17], C-reactive protein (CRP) level [18], E-selectin expression [19], and inflammatory disease such as aggressive peritonitis [20,21]. In addition, Fpr1 SNP (rs1042229) was also associated with stomach cancer [22]. However, the function of Fpr1 SNPs in human colorectal cancer has not been investigated.

In this study, we enrolled forty-eight Chinese CRC patients. Seven Fpr1 SNPs (c.289C>A, c.301G>C, c.306T $>$ C, c. $546 \mathrm{C}>\mathrm{A}$, c. $568 \mathrm{~A}>\mathrm{T}$, c. $576 \mathrm{~T}>\mathrm{C}>\mathrm{G}$, and c.1037C $>$ A) were detected. The allele and genotype frequencies of these SNPs in CRC tissues were not significantly different from that in whole blood cells derived from healthy Chinese subjects. We further examined the correlation between Fpr1 SNPs with clinicopathological characteristics and several specific diagnostic markers of CRC.

\section{Materials and methods}

\section{Human subjects}

A total of 60 surgically resected CRC tissue specimens were obtained from Changzheng Hospital, Shanghai, China. Histological classification and stage of the CRC were determined according to the UICC TNM classification system [23]. Acquisition of tissue specimens was approved by the Changzheng Hospital Institution Review Board of and was carried out under guidance of established ethical protocols. Patients with colorectal cancer were between 22 and 83 years old, and colonoscopy and pathological examinations were confirmed. Exclusion criteria: (1) with a history of other tumor diseases, or with other genetic diseases; (2) severe colorectal bleeding occurred within 30 days; (3) participating in other clinical trials requiring drugs within 60 days; (4) serious cardiovascular diseases, uncontrolled infections, or other uncontrolled co-existing diseases; (5) pregnant women; (6) subjects receiving preoperative neo-adjuvant therapies and/or radiotherapy. Each patient signed the informed consent, which outlined the purpose and application of the colorectal tumor tissue that they donated. All samples were fixed with $4 \%$ paraformaldehyde and cryoprotected in $30 \%$ sucrose for immunohistochemical staining or were kept frozen at $-80^{\circ} \mathrm{C}$ for other biochemistry analysis.

\section{DNA isolation, Polymerase Chain Reaction (PCR) and sequencing}

Genomic DNA was isolated from the CRC tissue specimens with a DNA isolation kit (GIAGEN, Hilden, Germany). To examine Fpr1 SNPs c. $289 \mathrm{C}>\mathrm{A}$, c. $301 \mathrm{G}>\mathrm{C}$, c. $306 \mathrm{~T}>\mathrm{C}$, c. $546 \mathrm{C}>\mathrm{A}$, c. $568 \mathrm{~A}>\mathrm{T}$, c.576T $>C>G$, and c.1037C $>A$, primers were designed to amplify a 1538bp fragment of the FPR1 gene containing these SNPs. The forward primer was 5'-TTGCCCAACAGGTACAATAA-3' and the reverse primer was 5'-ATTTCAGGCAAACTAGGATG-3'. The products were sequenced with the primers 5'-AGAACCACCGCACCGTGA-3' and 5'-GGATGTT CCGGCTGTTGT-3'. PCR was performed according to the following conditions: $95^{\circ} \mathrm{C}$ for $3 \mathrm{~min}, 40$ cycles of denaturation at $95^{\circ} \mathrm{C}$ for $20 \mathrm{~s}$, annealing at $56^{\circ} \mathrm{C}$ for 45 $\mathrm{s}$, and extension at $72{ }^{\circ} \mathrm{C}$ for $20 \mathrm{~s}$ with a final extension at $72{ }^{\circ} \mathrm{C}$ for $10 \mathrm{~min}$. All samples yielded PCR products, which were sequenced by Shanghai DNA biotechnologies.

\section{Immunohistochemistry}

The primary antibodies were mouse anti-human P53 protein and anti-human Ki67 protein (1:50; Santa Cruz Biotechnology, Santa Cruz, CA, United States), and rabbit anti-human CEA protein and rabbit monoclonal anti-HER2 protein (1:100; IBL, Tokyo, Japan). After incubation with the primary antibody, the sections were incubated with the secondary antibody and avidin-biotin-peroxidase complex. The slides were counterstained with hematoxylin and eosin. Positivity for P53 and Ki67 were defined as nuclear staining in $>10 \%$ of the tumor cell nuclei. Positivity for CEA was $>10 \%$ cytoplasmic staining. HER-2 immunostaining was scored as follows: 0-no reactivity or membranous reaction in fewer than $10 \%$ of cells, (1+)-faint complete or partial membranous reactivity in more than $10 \%$ of cells, $(2+)$-moderate complete or basolateral membranous reactivity in more than $10 \%$ of cells, and (3+)-strong complete or basolateral membranous reactivity in more than $10 \%$ of cells. The level of HER2 membranous expression was considered positive if IHC staining was $2+$ or $3+$. Immunohistochemical reactivity was interpreted blindly by two independent investigators.

\section{Data analysis}

Statistical analysis was performed using the statistic software GraphPad Prism 5 (San Diego, CA). Correlations between Fpr1 SNPs and various clinicopathological characteristics and specific diagnostic markers of CRC were analyzed by the Chi-square test. $\mathrm{P}$ values less than 0.05 was considered statistically significant. 


\section{Results}

\section{Basic clinical characteristics of the colorectal cancer patients}

In this study, we enrolled 60 Chinese CRC patients, with $30(50 \%)$ male and $30(50 \%)$ female. Their age ranged from 22 to 83 years (mean age, 54.5 years). 36 patients had colon cancer and 24 patients had rectal cancer. All patients had T0-T4 cancers according to the TNM classification. 29 patients were classified as highly or poorly differentiated and 31 as well or moderately differentiated. Ten of these patients had distant metastasis. The basic clinical characteristics of these patients are listed in Table 3.

\section{Single nucleotide polymorphisms of Fprl in Chinese colorectal cancer patients}

Seven previously identified Fpr1 SNPs (c.289C>A, c.301G>C, c.306T>C, c.546C>A, c.568A>T,
c.576T $>\mathrm{C}>\mathrm{G}$, and c.1037C $>\mathrm{A}$ ) were detected in Chinese CRC patients. The c.306T $>C$ and c.546C $>A$ are synonymous SNPs and other five sites are nonsynonymous SNPs. They are all located within exon 2 of the Fpr1 gene. The allele and genotype frequencies of these SNPs identified by sequence analysis of Fpr1 gene are demonstrated in Table 1 and 2. The minor allele frequency (MAF) for c.289A (p.L97M), c.301C (p.V101L), c.306C (p.F102F), c.546A (p.P182P), c.568T (p.R190W), and c.1037A (p.A346E) were 0.115, 0.448, $0.031,0.500,0.135$, and 0.344 respectively. The allele and genotype frequencies of these SNPs in CRC tissues were not significantly different from that in whole blood cells derived from healthy Chinese subjects. Data of healthy Chinese subjects are from Zhou's reports [24] and the HapMap resource (CHB, Han Chinese in Beijing, and CHS, Southern Han Chinese at https://www.ncbi.nlm.nih.gov/ variation/tools/1000genomes/).

Table 1. Allele frequencies in the CRC patients studied

\begin{tabular}{|c|c|c|c|c|c|c|c|}
\hline Reference ID & SNP & Allele & Phenotype & Frequency & & & \\
\hline & & & & Chinese CRC patients & $\begin{array}{l}\mathrm{CHB}^{*} \text { (Han Chinese in } \\
\text { Beijing) }\end{array}$ & $\begin{array}{l}\text { CHS* (Southern Han } \\
\text { Chinese) }\end{array}$ & $\mathrm{CH}^{\#}$ (Han Chinese) \\
\hline \multirow[t]{2}{*}{ rs78488639 } & L97M & c. $289 \mathrm{C}$ & Leu $^{97}$ & 0.885 & 0.918 & 0.938 & None \\
\hline & & c. $289 \mathrm{~A}$ & Met $^{97}$ & 0.115 & 0.082 & 0.062 & \\
\hline \multirow[t]{2}{*}{ rs2070745 } & V101L & c. $301 G$ & $\mathrm{Val}^{101}$ & 0.552 & 0.549 & 0.529 & 0.550 \\
\hline & & c.301C & Leu $^{101}$ & 0.448 & 0.451 & 0.471 & 0.450 \\
\hline \multirow[t]{2}{*}{ rs28930680 } & $\mathrm{F} 102 \mathrm{~F}$ & c.306T & Phe ${ }^{102}$ & 0.969 & 0.971 & 0.981 & None \\
\hline & & c. $306 \mathrm{C}$ & Phe ${ }^{102}$ & 0.031 & 0.029 & 0.019 & \\
\hline \multirow[t]{2}{*}{ rs2070746 } & P182P & c. $546 \mathrm{C}$ & Pro $^{182}$ & 0.500 & 0.524 & 0.486 & None \\
\hline & & c. $546 \mathrm{~A}$ & Pro 182 & 0.500 & 0.476 & 0.514 & \\
\hline \multirow[t]{2}{*}{ rs5030880 } & R190W & c. $568 \mathrm{~A}$ & Arg190 & 0.865 & 0.791 & 0.824 & 0.828 \\
\hline & & c.568T & $\operatorname{Tr} p^{190}$ & 0.135 & 0.209 & 0.176 & 0.172 \\
\hline \multirow[t]{3}{*}{ rs1042229 } & N192K & c.576T & Asn $^{192}$ & 0.521 & 0.524 & 0.567 & 0.514 \\
\hline & & c. $576 \mathrm{G}$ & Lys $^{192}$ & 0.344 & 0.262 & 0.262 & 0.304 \\
\hline & & c. $576 \mathrm{C}$ & Asn $^{192}$ & 0.135 & 0.214 & 0.171 & 0.182 \\
\hline \multirow[t]{2}{*}{ rs867228 } & $\mathrm{A} 346 \mathrm{E}$ & c.1037C & $\mathrm{Ala}^{346}$ & 0.656 & 0.714 & 0.705 & 0.703 \\
\hline & & c.1037A & Glu $^{346}$ & 0.344 & 0.286 & 0.295 & 0.297 \\
\hline
\end{tabular}

*Data from the HapMap resource (https://www.ncbi.nlm.nih.gov/variation/tools/1000genomes/); *Data from Zhou's report ${ }^{[24]}$.

Table 2. Genotype frequencies in the CRC patients studied

\begin{tabular}{lllll}
\hline Reference ID & SNP & Genotype & Frequency & \\
\cline { 3 - 4 } & & & Chinese CRC patients & CH (Han Chinese) \\
\hline rs78488639 & L97M & L/L & 0.792 & \\
& & L/M & 0.187 & 0.297 \\
rs2070745 & M/M & 0.021 & 0.507 \\
& V101L & V/V & 0.271 & 0.196 \\
& & V/L & 0.562 & None \\
rs28930680 & L/L & 0.167 & None \\
rs2070746 & F102F & F/F & 1.000 & 0.689 \\
rs5030880 & P182P & P/P & 1.000 & 0.278 \\
& R190W & R/R & 0.729 & 0.033 \\
rs1042229 & & R/W & 0.271 & 0.469 \\
& & W/W & 0.000 & 0.455 \\
rs867228 & N192K & N/N & 0.458 & 0.077 \\
& & N/K & 0.396 & 0.507 \\
& K346E & K/K & 0.146 & 0.392 \\
& & A/E & 0.396 & 0.100 \\
\hline
\end{tabular}

\#Data from Zhou's report ${ }^{[24]}$. 


\section{Correlation of Fprl SNPs with clinicopathological characteristics}

Next, we examined the correlation between Fpr1 SNPs with clinicopathological characteristics which included sex, age, AJCC stage and location of tumor, tumor size, differentiation, lymphatic invasion, distant metastasis, and pathological type. The significance of association between each of the SNPs and clinicopathological characteristics was determined by Chi-square test. Significant associations were observed between genotypes of c. $289 \mathrm{C}>\mathrm{A}$ and distant metastasis $(\mathrm{P}=0.001$, Table 3$)$. Also, significant associations were observed between genotypes of c.306T $>C$ and tumor size $(\mathrm{P}=0.016$, Table 3). Genotypes of c.546C $>\mathrm{A}$ was closer to tumor size and lymphatic invasion $(\mathrm{P}=0.012$ and $\mathrm{P}=0.043$, respectively, Table 4). Meanwhile, genotypes of c.1037C $>$ A was related with tumor location and differentiation $(\mathrm{P}=0.000$ and $\mathrm{P}=0.005$, respectively, Table 4). Besides, genotypes of c.576T $>C>G$ was related with pathological type $(\mathrm{P}=0.000$, Table 5$)$.

Furthermore, by using immunohistochemistry, we investigated some specific diagnostic markers of CRC such as P53, CEA, HER2, and Ki-67. The correlation between Fpr1 SNPs with these markers was analyzed (Table 6). The results showed that several Fpr1 SNP positions including c.289 (C>A) and c.576 $(\mathrm{G}>\mathrm{C}>\mathrm{T})$ were related to the expression of $\mathrm{P} 53$ $(\mathrm{P}=0.004$ and $\mathrm{P}=0.008$, respectively), and c.289 $(\mathrm{C}>\mathrm{A})$ and c.306T $>C$ to CEA $(P=0.03$ and $P=0.011$, respectively, Table 6). Otherwise, genotypes of c.576T $>\mathrm{C}>\mathrm{G}$ were also associated with HER2 $(\mathrm{P}=0.001$, Table 6). Besides, genotypes of c.289 (C>A) and c.576T $>C>G$ were related with Ki-67 $(P=0.034$ and $\mathrm{P}=0.001$, respectively, Table 6).

\section{Discussion}

Colorectal cancer, a highly invasive carcinoma, has high rates of venous invasion, lymphatic duct invasion, and lymph node metastasis [25]. Metastatic colorectal cancer (mCRC) is the main cause of the death in CRC patients [25]. In our previous study, the expression of FPR1 has been identified to be related to tumor serosal invasion in CRC patients. FPR1 activation promoted the migration and invasion of CRC cells [16]. In this work, we found the patients with Fpr1 homozygous 289A genotype showed a higher risk to distant metastasis than that with the $289 \mathrm{C} / \mathrm{C}$ or $289 \mathrm{~A} / \mathrm{C}$ genotypes $(\mathrm{P}=0.001)$, suggesting an association between Fpr1 genotype c.289 and the distant metastasis. In recent years, a large number of metastasis-associated genes have been identified. Via genomic DNA microarray and function annotation, Cai et al. reported that multiple SNP-associated genes specific to CRC metastasis were related to adhesion and immunity, such as cell motion, cell-substrate adhesion, regulation of innate immune response, etc [26]. Furthermore, many SNP-associated genes were also associated with adhesion- and immune-related signaling pathways, including adherens junction, chemokine signaling pathway, and cytokine-cytokine receptor interaction [26]. Otherwise, Lan and colleagues demonstrated that genetic polymorphisms of insulin-like growth factor 1 (IGF-1) and interleukin-8 (IL-8) increased susceptibility to lymph node metastasis in CRC patients [27]. All these data suggested that the polymorphisms of those immunerelated genes play an important role in the metastasis of CRC.

Table 3. Fprl SNPs with clinicopathological characteristics (c.289, c.301, and c.306)

\begin{tabular}{|c|c|c|c|c|c|c|c|c|c|c|c|c|}
\hline \multirow[t]{2}{*}{ Parameters } & \multicolumn{3}{|c|}{ Mutation of 289} & \multirow[t]{2}{*}{$P$ value } & \multicolumn{3}{|c|}{ Mutation of 301} & \multirow[t]{2}{*}{$P$ value } & \multicolumn{3}{|c|}{ Mutation of 306} & \multirow[t]{2}{*}{$p$ value } \\
\hline & $\mathrm{C} / \mathrm{C}$ & $\mathrm{C} / \mathrm{A}$ & $\mathrm{A} / \mathrm{A}$ & & $\mathrm{G} / \mathrm{G}$ & $\mathrm{G} / \mathrm{C}$ & $\mathrm{C} / \mathrm{C}$ & & $\mathrm{T} / \mathrm{T}$ & $\mathrm{T} / \mathrm{C}$ & $\mathrm{C} / \mathrm{C}$ & \\
\hline \multicolumn{13}{|l|}{ Gender } \\
\hline Female & 25 & 4 & 1 & 0.710 & 4 & 22 & 4 & 0.008 & 26 & 4 & 0 & 1.000 \\
\hline Male & 23 & 4 & 3 & & 11 & 10 & 9 & & 27 & 3 & 0 & \\
\hline \multicolumn{13}{|l|}{ Age (years) } \\
\hline$\leq 60$ & 26 & 4 & 2 & 1.000 & 5 & 22 & 5 & 0.042 & 29 & 3 & 0 & 0.695 \\
\hline$>60$ & 22 & 4 & 2 & & 10 & 10 & 8 & & 24 & 4 & 0 & \\
\hline \multicolumn{13}{|l|}{ AJCC } \\
\hline 0 -II & 24 & 6 & 0 & 0.057 & 6 & 18 & 6 & 0.621 & 23 & 7 & 0 & 0.011 \\
\hline III-IV & 24 & 2 & 4 & & 9 & 14 & 7 & & 30 & 0 & 0 & \\
\hline \multicolumn{13}{|c|}{ Tumor location } \\
\hline Colon & 26 & 7 & 3 & 0.170 & 7 & 21 & 8 & 0.474 & 30 & 6 & 0 & 0.225 \\
\hline Rectum & 22 & 1 & 1 & & 8 & 11 & 5 & & 23 & 1 & 0 & \\
\hline \multicolumn{13}{|c|}{ Size of tumor } \\
\hline$\leq 5$ & 29 & 5 & 0 & 0.064 & 6 & 24 & 4 & 0.198 & 27 & 7 & 0 & 0.016 \\
\hline$>5$ & 19 & 3 & 4 & & 9 & 18 & 9 & & 26 & 0 & 0 & \\
\hline \multicolumn{13}{|c|}{ Differentiation } \\
\hline I-II & 25 & 2 & 4 & 0.057 & 4 & 23 & 4 & 0.004 & 27 & 4 & 0 & 1.000 \\
\hline III-IV & 23 & 6 & 0 & & 11 & 9 & 9 & & 26 & 3 & 0 & \\
\hline Lymphatic & & & & & & & & & & & & \\
\hline
\end{tabular}




\begin{tabular}{|c|c|c|c|c|c|c|c|c|c|c|c|c|}
\hline \multirow{2}{*}{ Parameters } & \multicolumn{3}{|c|}{ Mutation of 289} & \multirow[t]{2}{*}{$P$ value } & \multicolumn{3}{|c|}{ Mutation of 301} & \multirow{2}{*}{$P$ value } & \multicolumn{3}{|c|}{ Mutation of 306} & \multirow[t]{2}{*}{$p$ value } \\
\hline & $\mathrm{C} / \mathrm{C}$ & $\mathrm{C} / \mathrm{A}$ & $\mathrm{A} / \mathrm{A}$ & & $\mathrm{G} / \mathrm{G}$ & $\mathrm{G} / \mathrm{C}$ & $\mathrm{C} / \mathrm{C}$ & & $\mathrm{T} / \mathrm{T}$ & $\mathrm{T} / \mathrm{C}$ & $\mathrm{C} / \mathrm{C}$ & \\
\hline Yes & 21 & 6 & 1 & 0.202 & 5 & 19 & 4 & 0.108 & 24 & 4 & 0 & 0.695 \\
\hline No & 27 & 2 & 3 & & 10 & 13 & 9 & & 29 & 3 & 0 & \\
\hline \multicolumn{13}{|l|}{ Distant Metastasis } \\
\hline Yes & 5 & 1 & 4 & 0.001 & 2 & 8 & 0 & 0.121 & 7 & 3 & 0 & 0.083 \\
\hline No & 43 & 7 & 0 & & 13 & 24 & 13 & & 46 & 4 & 0 & \\
\hline \multicolumn{13}{|l|}{ Pathological Type } \\
\hline Adenocarcinoma & 35 & 6 & 2 & 0.659 & 8 & 25 & 10 & 0.222 & 39 & 4 & 0 & 0.393 \\
\hline Mucinous & 13 & 2 & 2 & & 7 & 7 & 3 & & 14 & 3 & 0 & \\
\hline
\end{tabular}

Table 4. Fprl SNPs with clinicopathological characteristics (c.546, c.568, and c.1037)

\begin{tabular}{|c|c|c|c|c|c|c|c|c|c|c|c|c|}
\hline \multirow[t]{2}{*}{ Parameters } & \multicolumn{3}{|c|}{ Mutation of 546} & \multirow[t]{2}{*}{$P$ value } & \multicolumn{3}{|c|}{ Mutation of 568} & \multirow[t]{2}{*}{$p$ value } & \multicolumn{3}{|c|}{ Mutation of 1037} & \multirow[t]{2}{*}{$p$ value } \\
\hline & $\mathrm{C} / \mathrm{C}$ & $\mathrm{C} / \mathrm{A}$ & $\mathrm{A} / \mathrm{A}$ & & $\overline{G / G}$ & $\mathrm{G} / \mathrm{C}$ & $\mathrm{C} / \mathrm{C}$ & & $\mathrm{T} / \mathrm{T}$ & $\mathrm{T} / \mathrm{C}$ & $\mathrm{C} / \mathrm{C}$ & \\
\hline \multicolumn{13}{|l|}{ Gender } \\
\hline Female & 7 & 15 & 8 & 1.000 & 20 & 10 & 0 & 1.000 & 11 & 15 & 4 & 1.000 \\
\hline Male & 8 & 15 & 7 & & 21 & 9 & 0 & & 12 & 14 & 4 & \\
\hline \multicolumn{13}{|l|}{ Age (years) } \\
\hline$\leq 60$ & 5 & 17 & 10 & 0.206 & 27 & 5 & 0 & 0.006 & 15 & 12 & 5 & 0.218 \\
\hline$>60$ & 10 & 13 & 5 & & 14 & 14 & 0 & & 8 & 17 & 3 & \\
\hline \multicolumn{13}{|l|}{ AJCC } \\
\hline 0 -II & 9 & 12 & 9 & 0.283 & 21 & 9 & 0 & 1.000 & 10 & 17 & 3 & 0.514 \\
\hline III-IV & 6 & 18 & 6 & & 20 & 10 & 0 & & 13 & 12 & 5 & \\
\hline \multicolumn{13}{|l|}{ Tumor location } \\
\hline Colon & 8 & 18 & 10 & 0.742 & 24 & 12 & 0 & 0.784 & 19 & 17 & 0 & 0.000 \\
\hline Rectum & 7 & 12 & 5 & & 17 & 7 & 0 & & 4 & 12 & 8 & \\
\hline \multicolumn{13}{|l|}{ Size of tumor } \\
\hline$\leq 5$ & 8 & 22 & 4 & 0.012 & 32 & 2 & 0 & 0.000 & 12 & 18 & 4 & 0.716 \\
\hline$>5$ & 7 & 8 & 11 & & 9 & 17 & 0 & & 11 & 11 & 4 & \\
\hline \multicolumn{13}{|l|}{ Differentiation } \\
\hline I-II & 6 & 20 & 5 & 0.061 & 29 & 2 & 0 & 0.000 & 13 & 18 & 0 & 0.005 \\
\hline III-IV & 9 & 10 & 10 & & 12 & 17 & 0 & & 10 & 11 & 8 & \\
\hline \multicolumn{13}{|c|}{ Lymphatic invasion } \\
\hline Yes & 7 & 18 & 3 & 0.043 & 20 & 8 & 0 & 0.782 & 12 & 12 & 4 & 0.724 \\
\hline No & 8 & 12 & 12 & & 21 & 11 & 0 & & 11 & 17 & 4 & \\
\hline \multicolumn{13}{|l|}{ Distant Metastasis } \\
\hline Yes & 4 & 4 & 2 & 0.550 & 7 & 3 & 0 & 1.000 & 6 & 4 & 0 & 0.241 \\
\hline No & 11 & 26 & 13 & & 34 & 16 & 0 & & 17 & 25 & 8 & \\
\hline \multicolumn{13}{|l|}{ Pathological Type } \\
\hline Adenocarcinoma & 11 & 23 & 9 & 0.562 & 36 & 7 & 0 & 0.000 & 19 & 20 & 4 & 0.208 \\
\hline Mucinous & 4 & 7 & 6 & & 5 & 12 & 0 & & 4 & 9 & 4 & \\
\hline
\end{tabular}

Table 5. Fprl SNPs with clinicopathological characteristics (c.576)

\begin{tabular}{|c|c|c|c|c|c|c|c|}
\hline \multirow[t]{2}{*}{ Parameters } & \multicolumn{6}{|c|}{ Mutation of $576 \mathrm{n}(\%)$} & \multirow{2}{*}{$\begin{array}{l}p \\
\text { value }\end{array}$} \\
\hline & $\mathrm{T} / \mathrm{T}$ & $\mathrm{T} / \mathrm{C}$ & $\mathrm{T} / \mathrm{G}$ & $\mathrm{C} / \mathrm{C}$ & $\mathrm{C} / \mathrm{G}$ & $\mathrm{G} / \mathrm{G}$ & \\
\hline \multicolumn{8}{|l|}{ Gender } \\
\hline Female & 9 & 6 & 9 & 0 & 4 & 2 & 0.463 \\
\hline Male & 7 & 5 & 9 & 0 & 2 & 7 & \\
\hline \multicolumn{8}{|l|}{ Age (years) } \\
\hline$\leq 60$ & 12 & 2 & 10 & 0 & 4 & 4 & 0.053 \\
\hline$>60$ & 4 & 9 & 8 & 0 & 2 & 5 & \\
\hline \multicolumn{8}{|l|}{ AJCC } \\
\hline 0 -II & 9 & 5 & 6 & 0 & 3 & 7 & 0.282 \\
\hline III-IV & 7 & 6 & 12 & 0 & 3 & 2 & \\
\hline \multicolumn{8}{|c|}{ Tumor location } \\
\hline Colon & 9 & 7 & 12 & 0 & 3 & 5 & 0.937 \\
\hline Rectum & 7 & 4 & 6 & 0 & 3 & 4 & \\
\hline \multicolumn{8}{|c|}{ Size of tumor } \\
\hline$\leq 5$ & 8 & 6 & 12 & 0 & 2 & 6 & 0.628 \\
\hline$>5$ & 8 & 5 & 6 & 0 & 4 & 3 & \\
\hline \multicolumn{8}{|c|}{ Differentiation } \\
\hline I-II & 9 & 6 & 9 & 0 & 3 & 4 & 0.982 \\
\hline III-IV & 7 & 5 & 9 & 0 & 3 & 5 & \\
\hline Lymphatic & & & & & & & \\
\hline
\end{tabular}




\begin{tabular}{|c|c|c|c|c|c|c|c|}
\hline \multirow[t]{2}{*}{ Parameters } & \multicolumn{6}{|c|}{ Mutation of $576 \mathrm{n}(\%)$} & \multirow{2}{*}{$\begin{array}{l}p \\
- \text { value }\end{array}$} \\
\hline & $\mathrm{T} / \mathrm{T}$ & $\mathrm{T} / \mathrm{C}$ & $\mathrm{T} / \mathrm{G}$ & $\mathrm{C} / \mathrm{C}$ & $\mathrm{C} / \mathrm{G}$ & $\mathrm{G} / \mathrm{G}$ & \\
\hline Yes & 6 & 6 & 10 & 0 & 3 & 3 & 0.734 \\
\hline No & 10 & 5 & 8 & 0 & 3 & 6 & \\
\hline \multicolumn{8}{|l|}{ Distant Metastasis } \\
\hline Yes & 2 & 2 & 2 & 0 & 2 & 2 & 0.688 \\
\hline No & 14 & 9 & 16 & 0 & 4 & 7 & \\
\hline \multicolumn{8}{|l|}{ Pathological Type } \\
\hline Adenocarcinoma & 14 & 9 & 5 & 0 & 6 & 9 & 0.000 \\
\hline Mucinous & 2 & 2 & 13 & 0 & 0 & 0 & \\
\hline
\end{tabular}

Table 6. Fprl SNPs with specific diagnostic markers of CRC

\begin{tabular}{|c|c|c|c|c|c|c|c|c|c|c|c|c|}
\hline \multirow{2}{*}{$\begin{array}{l}\text { Parameters } \\
\text { (Total mutations) }\end{array}$} & \multicolumn{2}{|l|}{ P53 } & \multirow[t]{2}{*}{$p$ value } & \multicolumn{2}{|c|}{ CEA } & \multirow[t]{2}{*}{$p$ value } & \multicolumn{2}{|c|}{ HER-2 } & \multirow[t]{2}{*}{$p$ value } & \multicolumn{2}{|c|}{ Ki-67 } & \multirow[t]{2}{*}{$p$ value } \\
\hline & Yes & No & & Yes & No & & Yes & No & & Yes & No & \\
\hline c.289 & & & 0.004 & & & 0.030 & & & 0.001 & & & 0.034 \\
\hline $\mathrm{C} / \mathrm{C}$ & 39 & 9 & & 43 & 5 & & 30 & 18 & & 30 & 18 & \\
\hline $\mathrm{C} / \mathrm{A}$ & 3 & 5 & & 5 & 3 & & 0 & 8 & & 8 & 0 & \\
\hline $\mathrm{A} / \mathrm{A}$ & 1 & 3 & & 2 & 2 & & 1 & 3 & & 4 & 0 & \\
\hline c.301 & & & 0.409 & & & 0.429 & & & 0.277 & & & 0.060 \\
\hline $\mathrm{G} / \mathrm{G}$ & 9 & 6 & & 11 & 4 & & 5 & 10 & & 14 & 1 & \\
\hline $\mathrm{G} / \mathrm{C}$ & 25 & 7 & & 28 & 4 & & 18 & 14 & & 20 & 12 & \\
\hline $\mathrm{C} / \mathrm{C}$ & 9 & 4 & & 11 & 2 & & 8 & 5 & & 8 & 5 & \\
\hline c.306 & & & 0.092 & & & 0.011 & & & 0.247 & & & 1.000 \\
\hline $\mathrm{T} / \mathrm{T}$ & 40 & 13 & & 47 & 6 & & 29 & 24 & & 37 & 16 & \\
\hline $\mathrm{T} / \mathrm{C}$ & 3 & 4 & & 3 & 4 & & 2 & 5 & & 5 & 2 & \\
\hline $\mathrm{C} / \mathrm{C}$ & 0 & 0 & & 0 & 0 & & 0 & 0 & & 0 & 0 & \\
\hline c.546 & & & 0.188 & & & 0.283 & & & 0.006 & & & 0.090 \\
\hline $\mathrm{C} / \mathrm{C}$ & 11 & 4 & & 12 & 3 & & 13 & 2 & & 7 & 8 & \\
\hline $\mathrm{C} / \mathrm{A}$ & 24 & 6 & & 27 & 3 & & 13 & 17 & & 24 & 6 & \\
\hline $\mathrm{A} / \mathrm{A}$ & 8 & 7 & & 11 & 4 & & 5 & 10 & & 11 & 4 & \\
\hline c.568 & & & 1.000 & & & 0.711 & & & 0.100 & & & 1.000 \\
\hline $\mathrm{G} / \mathrm{G}$ & 29 & 12 & & 35 & 6 & & 18 & 23 & & 29 & 12 & \\
\hline $\mathrm{G} / \mathrm{C}$ & 14 & 5 & & 15 & 4 & & 13 & 6 & & 13 & 6 & \\
\hline $\mathrm{C} / \mathrm{C}$ & 0 & 0 & & 0 & 0 & & 0 & 0 & & 0 & 0 & \\
\hline c.1037 & & & 0.851 & & & 0.714 & & & 0.936 & & & 0.676 \\
\hline $\mathrm{T} / \mathrm{T}$ & 17 & 6 & & 19 & 4 & & 11 & 12 & & 15 & 8 & \\
\hline $\mathrm{T} / \mathrm{C}$ & 21 & 8 & & 25 & 4 & & 16 & 13 & & 22 & 7 & \\
\hline $\mathrm{C} / \mathrm{C}$ & 5 & 3 & & 6 & 2 & & 4 & 4 & & 5 & 3 & \\
\hline c.576 & & & 0.008 & & & 0.557 & & & 0.001 & & & 0.011 \\
\hline $\mathrm{T} / \mathrm{T}$ & 7 & 9 & & 11 & 5 & & 5 & 11 & & 13 & 3 & \\
\hline $\mathrm{T} / \mathrm{C}$ & 10 & 1 & & 10 & 1 & & 8 & 3 & & 10 & 1 & \\
\hline$T / G$ & 14 & 4 & & 16 & 2 & & 5 & 13 & & 14 & 4 & \\
\hline $\mathrm{C} / \mathrm{C}$ & 0 & 0 & & 0 & 0 & & 0 & 0 & & 0 & 0 & \\
\hline $\mathrm{C} / \mathrm{G}$ & 3 & 3 & & 5 & 1 & & 4 & 2 & & 2 & 4 & \\
\hline $\mathrm{G} / \mathrm{G}$ & 9 & 0 & & 8 & 1 & & 9 & 0 & & 3 & 6 & \\
\hline
\end{tabular}

As we mentioned earlier, a major cause of death in colorectal patients is metastasis [25]. Early clinical trials suggest the role for personalized HER2-targeted therapy in mCRC [28]. HER2, also known as ERBB2 or proto-oncogene $\mathrm{Neu}$, is a member of the human epidermal growth factor receptor family. Amplification or over-expression of HER2 has been reported to be closely related to the development and progression in certain aggressive types of cancers including breast cancer and CRC $[29,30]$. HER2 is also a potential biomarker guiding chemotherapy in advanced colorectal cancer [31]. Our results showed that genotypes of c.576T $>\mathrm{C}>\mathrm{G}$ were also associated with HER2. Previous studies have found that multiple factors may influence the expression of HER2 [32]. Since Fpr1 SNPs are closely associated with HER1 expression in CRC patients, further experiments are needed to verify how Fpr1 SNPs regulate the expression of HER2. Besides, the relationship between Fpr1 SNPs and HER2-targeted therapy in mCRC can also be explored.

Multiple studies reported that the genetic polymorphisms are associated with the occurrence and progression of CRC $[33,34]$. In this work, we found the allele and genotype frequencies of Fpr1 SNPs in CRC tissues were not significantly different from that in whole blood cells derived from healthy Chinese subjects. This result suggested that the polymorphisms of Fpr1 may have no effect on the occurrence of CRC. However, Fpr1 genotypes of c. $289 \mathrm{C}>\mathrm{A}$, c.306T $>$ C, c.546C $>$ A, c. $576 \mathrm{~T}>\mathrm{C}>\mathrm{G}$, and c.1037C $>$ A showed significant associations with the 
tumor size in CRC patients, indicating their potential role in the progression of CRC. The polymorphism of c.576 was also reported to be associated with susceptibility to stomach cancer and periodontitis [21, 22]. These results suggest that Fpr1 polymorphisms may be closely related to the occurrence and/or development of gastrointestinal tumors.

CEA, specifically expressed in biliary and gastrointestinal epithelium, is a diagnostic marker for CRC [35]. Previous studies reported that the expression of CEA in CRC was related to survival [36]. The expression of CEA is frequently higher in poorly differentiated colon cancer [37]. In this study, we found that the genotypes of Fpr1 c.289 (C>A) and c.306T $>C$ were highly associated with CEA expression in CRC. Further experiments are needed to verify the mechanism by which Fpr1 SNPs regulates CEA expression.

Our previous study found that the expression of FPR1 was related to tumor serosal invasion in CRC patients. FPR1 activation promoted the migration and invasion of CRC cells [16]. In this work, we further identified that Fpr1 SNP was associated with the distant metastasis of CRC. Fpr1 SNPs were also associated with the expression of P53, CEA, HER2 and Ki67. This is the first study to evaluate polymorphisms of Fpr1 in CRC and find positive correlations. Further replication study with a larger sample size is required. Additionally, research on interaction mechanism between Fpr1 SNPs and the positive related factors is needed.

\section{Abbreviations}

FPR1: formyl peptide receptor 1; CRC: colorectal cancer; SNP: single nucleotide polymorphisms; CEA: carcino-embryonic antigen.

\section{Acknowledgements}

This work was supported by grants from National Natural Science Foundation of China (Grant 81870835 and Grant 81571027.

\section{Competing Interests}

The authors have declared that no competing interest exists.

\section{References}

1. Bray F, Ferlay J, Soerjomataram I, Siegel RL, Torre LA, Jemal A. Global cancer statistics 2018: GLOBOCAN estimates of incidence and mortality worldwide for 36 cancers in 185 countries. CA Cancer J Clin. 2018; 68: 394-424.

2. Valle L, de Voer RM, Goldberg Y, Sjursen W, Forsti A, Ruiz-Ponte C, et al. Update on genetic predisposition to colorectal cancer and polyposis. Mol Aspects Med. 2019.

3. Weitz J, Koch M, Debus J, Hohler T, Galle PR, Buchler MW. Colorectal cancer. Lancet. 2005; 365: 153-65.

4. Su N, Xu XY, Chen H, Gao WC, Ruan CP, Wang Q, et al. Increased expression of annexin A1 is correlated with K-ras mutation in colorectal cancer. Tohoku J Exp Med. 2010; 222: 243-50.
5. Calvert PM, Frucht H. The genetics of colorectal cancer. Ann Intern Med. 2002; 137: 603-12.

6. Zhu L, Wang R, Zhang L, Zuo C, Zhang R, Zhao S. rs187960998 polymorphism in miR-211 prevents development of human colon cancer by deregulation of 3'UTR in CHD5. Onco Targets Ther. 2019; 12: 405-12.

7. Shamoun L, Skarstedt M, Andersson RE, Wagsater D, Dimberg J. Association study on IL-4, IL-4Ralpha and IL-13 genetic polymorphisms in Swedish patients with colorectal cancer. Clin Chim Acta. 2018; 487: 101-6.

8. Ye RD, Boulay F, Wang JM, Dahlgren C, Gerard C, Parmentier M, et al. International Union of Basic and Clinical Pharmacology. LXXIII. Nomenclature for the formyl peptide receptor (FPR) family. Pharmacol Rev. 2009; 61: 119-61

9. Le Y, Murphy PM, Wang JM. Formyl-peptide receptors revisited. Trends Immunol. 2002; 23: 541-8.

10. Murphy PM. The molecular biology of leukocyte chemoattractant receptors. Annu Rev Immunol. 1994; 12: 593-633.

11. Becker EL, Forouhar FA, Grunnet ML, Boulay F, Tardif M, Bormann BJ, et al. Broad immunocytochemical localization of the formylpeptide receptor in human organs, tissues, and cells. Cell Tissue Res. 1998; 292: 129-35.

12. Prevete N, Liotti F, Illiano A, Amoresano A, Pucci P, de Paulis A, et al. Formyl peptide receptor 1 suppresses gastric cancer angiogenesis and growth by exploiting inflammation resolution pathways. Oncoimmunology. 2017; 6: e1293213.

13. de Paulis A, Prevete N, Rossi FW, Rivellese F, Salerno F, Delfino G, et al. Helicobacter pylori $\mathrm{Hp}(2-20)$ promotes migration and proliferation of gastric epithelial cells by interacting with formyl peptide receptors in vitro and accelerates gastric mucosal healing in vivo. J Immunol. 2009; 183: 3761-9.

14. Cattaneo F, Guerra G, Parisi M, Lucariello A, De Luca A, De Rosa N, et al. Expression of Formyl-peptide Receptors in Human Lung Carcinoma. Anticancer Res. 2015; 35: 2769-74.

15. Khau T, Langenbach SY, Schuliga M, Harris T, Johnstone CN, Anderson RL, et al. Annexin-1 signals mitogen-stimulated breast tumor cell proliferation by activation of the formyl peptide receptors (FPRs) 1 and 2. FASEB J. 2011; 25: 483-96.

16. Li SQ, Su N, Gong P, Zhang HB, Liu J, Wang D, et al. The Expression of Formyl Peptide Receptor 1 is Correlated with Tumor Invasion of Human Colorectal Cancer. Sci Rep. 2017; 7: 5918.

17. El Shamieh $S$, Herbeth $B$, Azimi-Nezhad $M$, Benachour $H$, Masson $C$, Visvikis-Siest S. Human formyl peptide receptor 1 C32T SNP interacts with age and is associated with blood pressure levels. Clin Chim Acta. 2012; 413: 34-8.

18. Bhattacharya M, Wang J, Ribeiro FM, Dixon SJ, Feldman RD, Hegele RA, et al. Analysis of a missense variant of the human $\mathrm{N}$-formyl peptide receptor that is associated with agonist-independent beta-arrestin association and indices of inflammation. Pharmacogenomics J. 2007; 7: 190-9.

19. Benachour H, Zaiou M, Herbeth B, Lambert D, Lamont JV, Pfister M, et al. Human formyl peptide receptor 1 (FPR1) c.32C $>\mathrm{T}$ SNP is associated with decreased soluble E-selectin levels. Pharmacogenomics. 2009; 10: 951-9.

20. Maney P, Walters JD. Formylpeptide receptor single nucleotide polymorphism $348 \mathrm{~T}>\mathrm{C}$ and its relationship to polymorphonuclear leukocyte chemotaxis in aggressive periodontitis. J Periodontol. 2009; 80: 1498-505.

21. Zhang Y, Syed R, Uygar C, Pallos D, Gorry MC, Firatli E, et al. Evaluation of human leukocyte N-formylpeptide receptor (FPR1) SNPs in aggressive periodontitis patients. Genes Immun. 2003; 4: 22-9.

22. Otani $\mathrm{T}$, Ikeda $\mathrm{S}$, Lwin $\mathrm{H}$, Arai $\mathrm{T}$, Muramatsu M, Sawabe M. Polymorphisms of the formylpeptide receptor gene (FPR1) and susceptibility to stomach cancer in 1531 consecutive autopsy cases. Biochem Biophys Res Commun. 2011; 405: 356-61.

23. Sobin LH, Fleming ID. TNM Classification of Malignant Tumors, fifth edition (1997). Union Internationale Contre le Cancer and the American Joint Committee on Cancer. Cancer. 1997; 80: 1803-4.

24. Zhou C, Zhou Y, Wang J, Feng Y, Wang H, Xue J, et al. V101L of human formyl peptide receptor 1 (FPR1) increases receptor affinity and augments the antagonism mediated by cyclosporins. Biochem J. 2013; 451: 245-55.

25. Cremolini C, Schirripa M, Antoniotti C, Moretto R, Salvatore L, Masi G, et al. First-line chemotherapy for mCRC-a review and evidence-based algorithm. Nat Rev Clin Oncol. 2015; 12: 607-19.

26. Cai Z, Han S, Li Z, He L, Zhou J, Huang W, et al. A genome-wide assessment of variations of primary colorectal cancer maintained in metastases. Gene. 2016; 595: 18-24.

27. Lan YT, Yang SH, Lin JK, Lin CC, Wang HS, Chen WS, et al. Genetic variations are associated with lymph node metastasis in colorectal cancer patients. J Surg Oncol. 2014; 110: 307-12.

28. Kraus S, Nabiochtchikov I, Shapira S, Arber N. Recent advances in personalized colorectal cancer research. Cancer Lett. 2014; 347: 15-21.

29. Slamon DJ, Leyland-Jones B, Shak S, Fuchs H, Paton V, Bajamonde A, et al. Use of chemotherapy plus a monoclonal antibody against HER2 for metastatic breast cancer that overexpresses HER2. N Engl J Med. 2001; 344: 783-92.

30. Song Z, Deng Y, Zhuang K, Li A, Liu S. Immunohistochemical results of HER2/neu protein expression assessed by rabbit monoclonal antibodies SP3 and 4B5 in colorectal carcinomas. Int J Clin Exp Pathol. 2014; 7: 4454-60.

31. Feng Y, Li Y, Huang D, Cai S, Peng J. HER2 as a potential biomarker guiding adjuvant chemotherapy in stage II colorectal cancer. Eur J Surg Oncol. 2019; 45: $167-73$ 
32. Singla H, Ludhiadch A, Kaur RP, Chander H, Kumar V, Munshi A. Recent advances in HER2 positive breast cancer epigenetics: Susceptibility and therapeutic strategies. Eur J Med Chem. 2017; 142: 316-27.

33. Abd El-Fattah AA, Sadik NAH, Shaker OG, Mohamed Kamal A. Single Nucleotide Polymorphism in SMAD7 and CHI3L1 and Colorectal Cancer Risk. Mediators Inflamm. 2018; 2018: 9853192.

34. Naccarati A, Pardini B, Hemminki K, Vodicka P. Sporadic colorectal cancer and individual susceptibility: a review of the association studies investigating the role of DNA repair genetic polymorphisms. Mutat Res. 2007; 635: 118-45.

35. Chiang JM, Hung HY, You JF, Chiang SF, Lee CF, Chou HS, Lee WC, Chan KM: Applicability of postoperative carcinoembryonic antigen levels in determining post-liver-resection adjuvant chemotherapy regimens for colorectal cancer hepatic metastasis. Medicine 2019, 98(44):e17696.

36. Kim JH, Jun $\mathrm{KH}$, Jung H, Park IS, Chin HM: Prognostic Value of Preoperative Serum Levels of Five Tumor Markers (Carcinoembryonic Antigen, CA19-9, Alpha-fetoprotein, CA72-4, and CA125) in Gastric Cancer. Hepato-gastroenterology 2014, 61(131):863-869.

37. Liu Q, Huang Y, Luo D, Zhang S, Cai S, Li Q, Ma Y, Li X: Evaluating the Guiding Role of Elevated Pretreatment Serum Carcinoembryonic Antigen Levels for Adjuvant Chemotherapy in Stage IIA Colon Cancer: A Large Population-Based and Propensity Score-Matched Study. Frontiers in oncology 2019, 9:37. 